\title{
Differential Expression of Kinases and Cell Cycle Proteins in EW
}

\author{
LFM Scinto ${ }^{1 *}$ and Changiz Geula ${ }^{2}$ \\ ${ }^{1}$ Cambridge Neuro diagnostics LTD, UK \\ ${ }^{2}$ Northwestern University Medical School, USA
}

Submission: December 20, 2016; Published: March 08, 2017

*Corresponding author: LFM Scinto, President, Cambridge Neurodiagnostics LTD, London, 15 Queen Anne's Grove London W4 1HW, England, UK, Tel: 1-617-407-0042; Email: leandor@cantab.net

\section{Abstract}

The Edinger-Westphal (EW) of the nucleus of cranial nerve III (NCNIII), in contrast to the somatic portion, is an early site of pathology and cell loss in Alzheimer's disease (AD) and the aging brain. Cell loss in the EW accounts for the observed pupillary hypersensitivity in Alzheimer's disease (AD) patients to challenge with dilute tropicamide that could potentially serve as a biomarker for AD. The remarkable specificity of pathophysiology and cell loss offers a possible heuristic model by which to study selective cell vulnerability. Neurochemical differences in the somatic and EW portions of the NCNIII may account for their differential vulnerability. The EW pathology consists of phosphorylated tau in oligomers, tangles and neurites. This suggests that neurochemicals shown to be critical to the metabolism and phosphorylation of tau may be differentially expressed at the protein level in these two cell groups. This study examined the cellular expression of Cdc2, Cdk5 kinases, the prolyl isomerase Pin 1 and mitotic phosphoepitopes visualized by MPM-2 using immunohistochemical methods. The results demonstrated that the expression of target neurochemicals differs in the EW as opposed to somatic cell groups and in AD patients versus controls. The variability in the expression of some of these proteins may account for the observed regionally specific pathophysiology and cell loss within the NCNIII and perhaps also other cell groups vulnerable in $\mathrm{AD}$ and ageing.

\section{Introduction}

Not all cells in the central nervous system (CNS) are vulnerable to the ravages of aging or degenerative disease (e.g. $\mathrm{AD}, \mathrm{PD}, \mathrm{ALS}$ ). Selective cell loss is a prominent and well noted feature of both aging and neurodegenerative disorders [1,2]. However, our understanding of the reasons for such selective vulnerability remains imperfect at best. Previous work in our Laboratory [3-5] has led to two observations that form the rationale for this study of the neurochemical basis for selective cell vulnerability in the brain:

A.Edinger-Westphal (EW) neurons of nucleus of cranial nerve III (NCNIII) in contrast to somatic neurons of this nucleus are vulnerable to $\mathrm{AD}$ pathology early in the course of the disease $[4,5]$.

Table 1: Consistent with this pathological vulnerability, the EW division of CNIII is subject to severe cell loss in AD patients.

\begin{tabular}{|c|c|c|c|c|}
\hline Case \# & Diagnosis & Age & Gender & PMI \\
\hline 1 & $\mathrm{AD}$ & 83 & $\mathrm{M}$ & 12 \\
\hline 2 & $\mathrm{AD}$ & 84 & $\mathrm{~F}$ & 13 \\
\hline 3 & $\mathrm{AD}$ & 81 & $\mathrm{~F}$ & 6 \\
\hline 4 & $\mathrm{AD}$ & 77 & $\mathrm{M}$ & 16 \\
\hline
\end{tabular}

\begin{tabular}{|c|c|c|c|c|}
\hline 5 & AD & 86 & F & 14 \\
\hline 6 & NC & 69 & M & 20 \\
\hline 7 & NC & 50 & F & 17 \\
\hline 8 & NC & 87 & F & 12 \\
\hline 9 & NC & 72 & M & 19 \\
\hline 10 & NC & 71 & F & 7 \\
\hline
\end{tabular}

B.Consistent with this pathological vulnerability, the EW division of CNIII is subject to severe cell loss in AD patients and clinically silent subjects with early tau pathology, while the somatic division exhibits no such loss [4] (Table 1).

The remarkable selectivity of pathology and cell loss in this well-defined small and compact nucleus offers an opportunity to study some of the possible underlying mechanisms for selective cell vulnerability in the CNS. The cells in the somatic and EW portions of this nucleus do not differ in their basic neurochemical characteristics [3] or in their developmental origin. They are both cholinergic cells groups enriched with the cholinergic enzymes choline acetyltransferase (ChAT) and acetylcholinesterase (AChE). While there likely are multiple paths and mechanisms leading to cell loss or synaptic dysfunction in aging and degenerative disease, abnormal tau 
phosphorylation is a significant and common feature of many degenerative diseases and is characteristic of the aging brain itself [5-9]. The pathology that is seen in the EW of AD cases is almost exclusively tau-based, with prominent numbers of phosphorylated tau-positive, dystrophic neurites, neuropil threads, tangles and pre-tangles (neurons without tangles which display accumulation of abnormally phosphorylated tau). This same tau pathology leading to cytoskeletal abnormalities is to a lesser extent a characteristic feature of the aging brain [7].

Tau is a microtubule-associated protein that binds to microtubules and stabilizes their organization within the axon and thus helps maintain axonal transport and contributes to cytoskeletal integrity [10]. In $\mathrm{AD}$, tau is phosphorylated at serine (Ser) or threonine (Thr) residues usually followed by a proline (Ser-Thr-Pro), although phosphorylation at these sites followed by another amino acid has also been described (SerThr-X). The phosphorylation of tau at many of these sites has been shown to inhibit its binding to microtubules, and thus leads to microtubule disorganization [10-12]. It has been suggested that unbound phosphorylated tau tends to aggregate, giving rise to the cytoskeletal abnormalities observed in a number of degenerative conditions (e.g. AD) as well as in the aging brain of non-demented individuals [11,13-15].

We hypothesized that the selective vulnerability of EW neurons to tau pathology compared to somatic neurons of the NCNIII might be due to a difference in the cellular expression of certain neurochemicals involved in the metabolism and phosphorylation cycle of tau. We specifically examined the cellular expression of Pin1, Cdk5, Cdc2, the Cdc2 activator cyclin B1 and the phosphoepitopes recognized by MPM2 in EW and somatic cells in the NCNIII. The choice of these proteins was motivated by the following findings: the observation that Pin 1 may play a significant role in the phosphorylation cycle of tau and in fact may restore the biological function of hyperphosphorylated tau $[10,16,17]$; the presence of Cdc2 and co-localization with PHF tau in cells in AD brain [18], suggested by some to indicate the re-entry of such cells into the cell cycle; the convincingly demonstrated role of the Cdc2 family of kinases and in particular Cdk5 in the phosphorylation cycle of tau [19]. Cdk5 has been shown to be a powerful pathological trigger for abnormal tau phosphorylation in AD brains [20,21].

Further it has been shown that Pin 1 exhibits high affinity binding to T231 phosphorylated tau. Research [10] has demonstrated that T231 phosphorylation is necessary for Pin 1 to bind to tau. T231 is readily phosphorylated by Cdc2 kinase. We hypothesize that in the absence of sufficient pools of Pin 1 , abundant pools of Cdc2 (or perhaps Cdk5, a member of the Cdc2 family) will lead to increased phosphorylation of tau at T231.

Cdc2 is a serine/threonine protein kinase composed of the catalytic subunit Cdc2 $(\sim 34 \mathrm{KDa})$ and its regulatory subunit cyclin B $(\sim 48 \mathrm{KDa})$. As a monomer, Cdc2 is inactive and requires association with cyclin B and phosphorylation of threonine 161. In mammalian cells, Cdc2 regulates entry into mitosis (transition from $\mathrm{G} 2$ to $\mathrm{M}$ phase) by phosphorylating key proteins including RNA polymerase II and histone. Cdc2 has been shown to phosphorylate tau at T231 [12]. Phosphorylation of tau by Cdc2 abolishes its ability to promote microtubule assembly.

To determine if the potentially active complex Cdc2/cyclin B1 was expressed in terminally differentiated neurons of the NCNIII we examined the expression of Cyclin B1 and MPM2 to visualize the generic mitotic epitopes produced by $\mathrm{Cdc} 2$ $[22,23]$. Using immunohistochemistry to characterize the cellular expression of the target neurochemicals it is possible to establish a typology of expression that may help to distinguish somatic cells from EW cells in the NCNIII and explain their differential vulnerability to tau hyperphosphorylation and cell death. If, as we hypothesize, there are significant differences in the cellular expression or location within the cellular matrix of some or all of the target neurochemicals, we will be able to pursue interventional studies (using organotypic slice cultures) to manipulate these neurochemicals to test their ability to inhibit tau hyperphosphorylation and cell loss or to protect cells from toxicity in aging and degenerative disease.

\section{Experimental Procedures}

\section{Cases}

We examined the NCNIII of five cases with clinically (NINCDS-ADRDA) [24] and pathologically-confirmed AD [25] and five normal age-matched elder controls free from cortical and midbrain pathology (Table 2).

Table 2: Pattern and Strength of Cdk5 expression in NCNIII in AD and Normal Cases.

\begin{tabular}{|c|c|c|c|}
\hline & Perinuclear & Nuclear & Soma \\
\hline Somatic-AD & ++ & \pm & + \\
\hline Somatic-NC & ++ & \pm & + \\
\hline EW-AD & ++ & ++ & \pm \\
\hline EW-NC & ++ & ++ & \pm \\
\hline
\end{tabular}

\section{+: Moderate}

++ : Strong

\pm : Weak

\section{Tissue preparation and neuropathological observations}

The brainstem of each case was separated by a cut placed rostral to substantia nigra, well into the thalamus, to preserve the entire oculomotor nucleus. Then the hemispheres were separated and the right hemisphere cut into $2-3 \mathrm{~cm}$ coronal slabs. All brain tissue were placed in cold $4 \%$ para formaldehyde in $0.1 \mathrm{M}$ phosphate buffer ( $\mathrm{pH} 7.4$ ) for 24-30 hours, then into 
graded concentrations of sucrose $(10-40 \%$ in $0.1 \mathrm{M}$ phosphate buffer) for cryoprotection and stored at $4{ }^{\circ} \mathrm{C}$.

All fixed tissue was sectioned at $40 \mu \mathrm{m}$ on a freezing microtome into $0.1 \mathrm{M}$ phosphate buffer containing $0.02 \%$ sodium azide. Adjacent series composed of 1 in 54 sections of the hemispheric blocks were used for pathological diagnosis. One in eight sections of the midbrain, the entorhinal block and the block from the central sulcus were collected for cytopathological and neurochemical studies.

\section{Pathological diagnosis and subject classification}

Adjacent series of sections from cortical blocks containing temporal, parietal, frontal and occipital cortex were stained with hematoxylin and eosin, Bielschowsky and thioflavin S methods. For the diagnosis of AD, senile plaques (SP) and neurofibrillary tangles (NFT) were counted in 10 random fields of cortical tissue and in hippocampus and amygdala and the mean SP and NFT counts per 200X optical field calculated. Only brains with numbers of SPs and NFTs in neocortex, hippocampus and amygdala characteristic of AD were accepted as coming from an $\mathrm{AD}$ patient, and only brains with no or scarce counts of cortical SPs and NFTs consistent with normal aging were considered normal [25]. Brains from clinically silent controls or those who exhibited only mild cognitive changes (consistent with published criteria for mild cognitive impairment) and who exhibited modest to moderate numbers of SPs and NFTs or that met CERAD criteria for possible AD but that did not meet pathological criteria for AD were excluded [26]. Any brains in which additional neuropathological features (such as significant cerebrovascular disease, vasculitis, etc.) were found were also excluded from this study.

\section{Antibodies and protein}

We used two antibodies to visualize tau pathology in tissue, Cdk5 (J3) a mouse monoclonal antibody from Santa Cruz and p35 (c-19), rabbit, also from Santa Cruz. For Pin 1 visualization we used a mouse monoclonal antibody Pin1(H-123) from Santa Cruz and a mouse monoclonal antibody developed by Dr. Ping Lu. We used three antibodies to study Cdc2 immunoreactivity and test the specificity of the reaction, two rabbit polyclonals, one from Abcam (Cdc2, p34) and one from Upstate and a mouse monoclonal antibody (anti-Cdc2) from Novus. MPM-2 and Cyclin B1 antibodies were obtained from Abcam. Purified Cdc2 protein for Western Blots was obtained from Abcam.

\section{Immuno histochemistry}

Immunohistochemistry was carried out using the avidinbiotin-peroxidase complex (ABC) method employing the vectastain Elite $\mathrm{ABC}$ kit (Vector Laboratories, Burlingame, CA). Free floating sections were rinsed $3 \mathrm{X}$ in $0.1 \mathrm{M}$ phosphate buffered saline (PBS), at $\mathrm{pH}$ 7.4. This rinse was repeated after every incubation step. The sections were treated with $0.4 \%$ triton X-100 in PBS for 30 minutes at room temperature and then soaked for 1 hour in the carrier medium consisting of $10 \%$ normal goat serum and $0.5 \%$ triton X-100 in PBS. The tissue was incubated in the primary antibody at appropriate dilutions for 24 hours at $4{ }^{\circ} \mathrm{C}$. Sections were then incubated in biotinylated goat secondary IgG (1/500) for 15 hours and then in the ABC complex (1/100) for 2 hours. The resultant peroxidase labeling was visualized by incubating the sections in $0.005 \%$ diaminobenzidine and $0.01 \% \mathrm{H} 2 \mathrm{O} 2$ in $50 \mathrm{mM}$ Tris- $\mathrm{HCl}(\mathrm{pH}$ 7.6) for 10-20 minutes. Following termination of the reaction by rinsing in the Tris- $\mathrm{HCl}$ buffer, the sections were mounted on slides, air-dried, dehydrated in graded alcohols, cleared in xylene and coverslipped under permount. Control sections were processed using nonspecific IgG in place of the primary antibody or by omitting the primary antibody. When excess antigens were available (see below for Cdc2) antibody was preabsorbed as a control. When done no staining was evident.

\section{Western blot}

Western blot analysis of Cdc2 expression was carried out on whole cell proteins extracted from the hippocampal formation of AD cases and analysed on 12\% SDS-PAGE and transferred to PVDF membrane. The membranes were probed with the above antibodies and immunoreactivity was visualized with peroxidase conjugated secondary antibody and ECL detection kit (Amersham). At dilutions of $1 / 1200$ to $1 / 1500$ of the primary antibody to Cdc2 the analysis was specific in recognizing the Cdc2 protein.

\section{Semi-quantitative assessment of the pattern and intensity of immunoreactivity}

To assess differences in expression, we created tables of the patterns of reactivity in specific structures (EW and Somatic) and in specific cellular compartments (soma, nucleus, perinuclear compartment). Patterns of reactivity were assessed by examining all sections from all cases that spanned the entire structure (anterior, medial and posterior). We also carried out a semi-quantitative analysis of the intensity of immunoreactivity for the patterns observed for Cdc2 and Pin 1. Kinase and protein expression in tissue were graded on the following scale based on visual inspection of all neurons in sections that spanned the structure and that contained somatic and EW neurons. This rating was carried out by three raters who were blind to diagnosis. Calculating Cronbach's alpha showed 94\% agreement between raters.

$$
\begin{aligned}
& 1=\text { no reactivity } \\
& 2 \text { = light reactivity } \\
& 3=\text { moderate reactivity } \\
& 4=\text { strong reactivity }
\end{aligned}
$$

Statistical analysis was carried out using non-parametric tests (Mann-Whitney U or Kolmogorov-Smirnov tests). 


\section{Open Access Journal of Neurology \& Neurosurgery}

Results

\section{Cdk5}

Table 3:Semi-quantative assessment OfPattern of Cdc2 Immunoreactivity in Somatic and EW regions of Normal and AD cases.

\begin{tabular}{|c|c|c|c|c|}
\hline Case & Somatic NC & $\begin{array}{c}\text { Somatic } \\
\text { AD }\end{array}$ & $\begin{array}{c}\text { Edinger- } \\
\text { WestphalNC }\end{array}$ & $\begin{array}{c}\text { Edinger- } \\
\text { Westphal } \\
\text { AD }\end{array}$ \\
\hline 1 & 3.5 & 2.9 & 1.6 & 1.9 \\
\hline 2 & 3 & 3 & 1.4 & 1.6 \\
\hline 3 & 3 & 3.3 & 1.3 & 1.4 \\
\hline 4 & 3.6 & 3.5 & 1.6 & 1.8 \\
\hline 5 & 3.4 & 3.2 & 1.4 & 1.3 \\
\hline
\end{tabular}

1: No reactivity

2: Light reactivity

3: Moderate reactivity

4: Strong reactivity

\section{4: Strong reactivity}

Cdk5 has been implicated in the abnormal phosphorylation of tau in $\mathrm{AD}$ [20]. It, unlike Cdc2, is expressed in terminally differentiated neurons and is activated in neurons by proteolytic cleavage of its neuron activator p35 to p25 [27]. Cdk5 immunoreactivity was present in abundance at the cellular level in neurons of both the somatic and EW portions of the NCNIII in normal and AD cases. In the somatic portion of normal control and $\mathrm{AD}$ cases it was located both in the soma of neurons as well as showing robust staining in the perinuclear region (Figure 1, panels A \& C). However, the EW of normal cases largely showed diffuse staining of the cell soma (Figure 1, panel B) (Table 3) whereas in the EW of AD cases (Figure 1, panel D), Cdk5 immunoreactivity appeared largely restricted to the perinuclear region and the nuclear compartment of neurons. Staining in the cell soma was significantly fainter compared to that seen in the somatic portion.

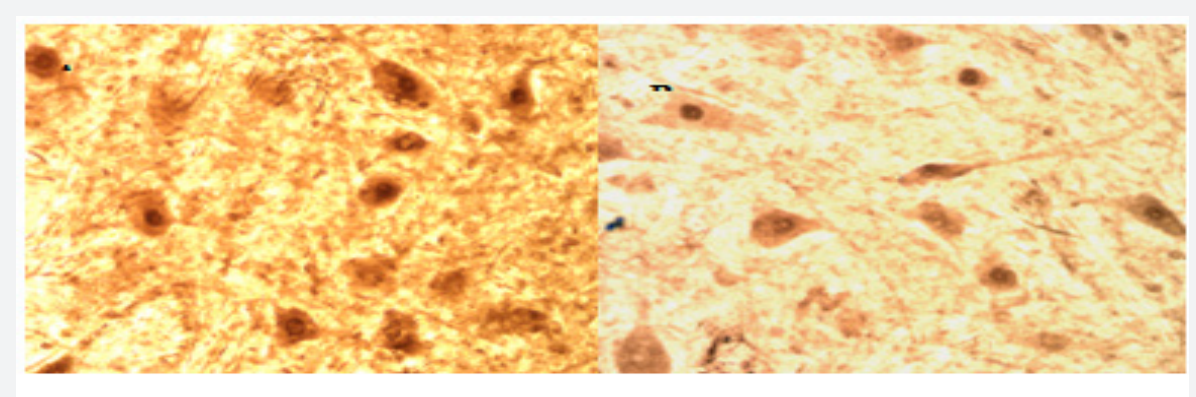

A

B

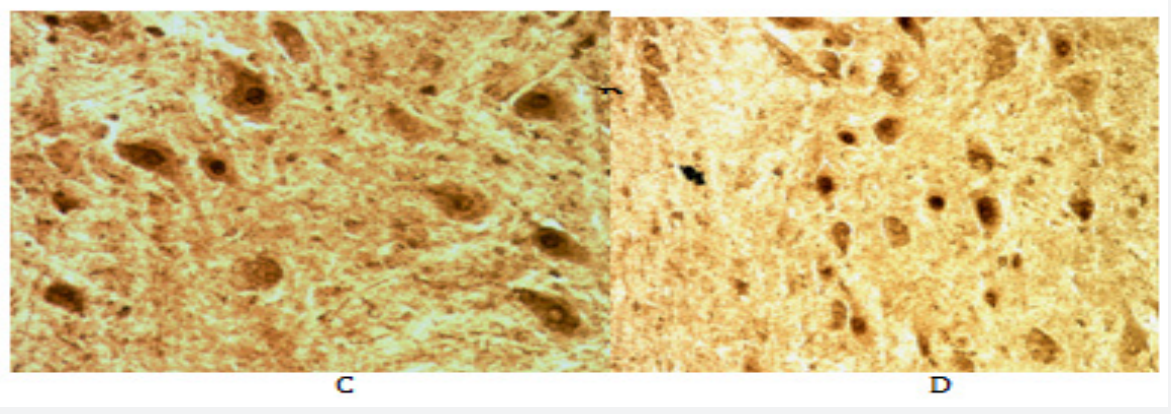

Figure 1: Panels $A \&$ \& are somatic cells, normal and $A D$ respectively; Panels $B$ \& $D$ are EW cells, normal and $A D$ respectively.

\section{Cdc2}

Cdc2 has been generally accepted to be up-regulated in mitotic cells but down-regulated in terminally differentiated cells such as neurons $[19,22,23,28,29]$. Some work has reported that $\mathrm{Cdc} 2$ seems to be present in degenerating neurons in AD and co-localizes with PH-tau $[18,29]$. This finding was interpreted as suggesting that degenerating cells in $\mathrm{AD}$ might attempt to reenter mitosis but then abort this process.

Contrary to expectations and reports suggesting otherwise, our observations revealed Cdc2 immunoreactivity to be strongly present in somatic neurons seemingly impervious to tau pathology in both control and AD cases (Figure 2). In contrast,
Cdc2 appeared to be only weakly expressed in EW neurons in normal and $\mathrm{AD}$ cases (Figure 2). To test the specificity of this finding we used three different antibodies to Cdc2 (one polyclonal and two monoclonal) and conducted a preabsorption assay using Cdc2 protein. All antibodies used resulted in the same pattern of staining. The preabsorption assay (Figure 3) showed abolition of virtually all staining for Cdc2.

Results of a Western blot indicated that at a dilution of $1 / 1200$ to $1 / 1500$ of the primary antibody to Cdc2 was specific in recognizing the Cdc2 protein (Figure 3, panel C). Lane 1 is protein extract from a young case, lane 2 is from an older case and lane 3 is purified Cdc2 protein. The results of a semi-quantitative analysis of the intensity of Cdc2 immunoreactivity are presented 


\section{Open Access Journal of Neurology \& Neurosurgery}

in Table 4. Overall for both AD and normal cases Cdc2 was more intensely expressed in the somatic neurons of NCNIII compared to EW neurons (Mann-Whitney U test, $\mathrm{p}=.000$ ). Further analysis revealed that there were no differences in intensity of expression of Cdc2 in somatic and EW neurons for AD and normal cases (Two-Sample Kolmogorov-Smirnov test: somatic, $\mathrm{p}=.819$; EW, $\mathrm{p}=.819)$.

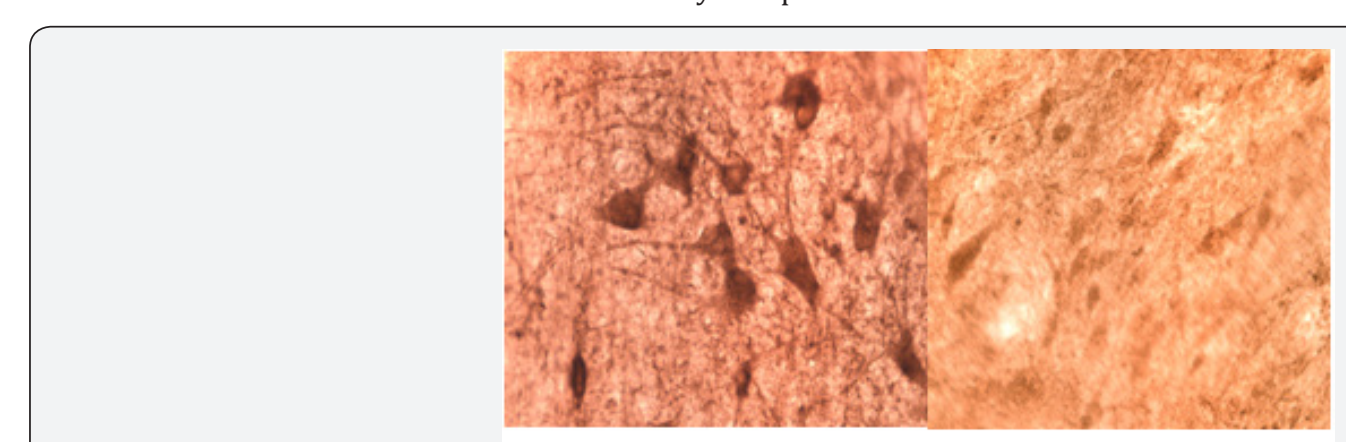

A

B

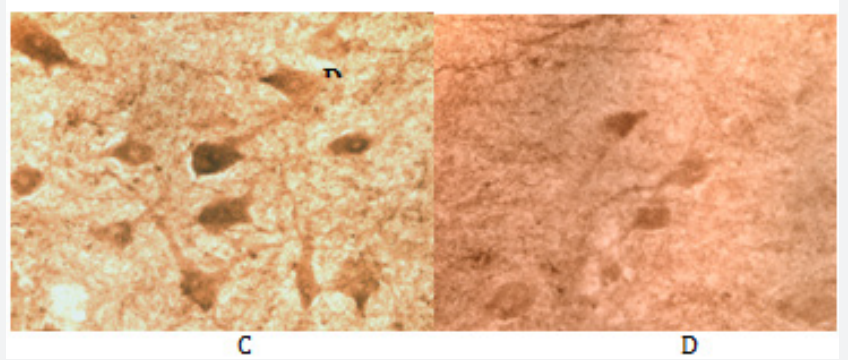

Figure 2: Panels $A \& C$ are normal case and $A D$ case somatic cells, respectively; Panels $B$ \& $D$ are normal case and $A D$ case $E W$ cells, respectively.

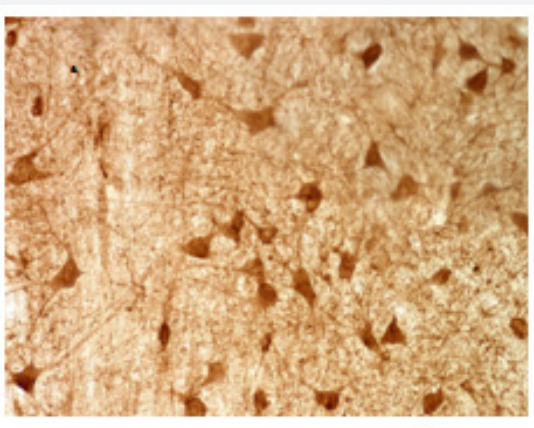

A

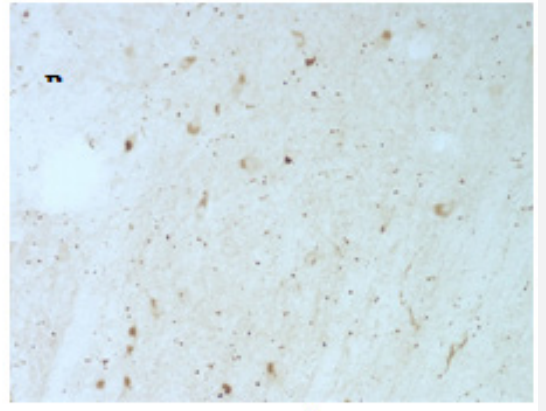

B

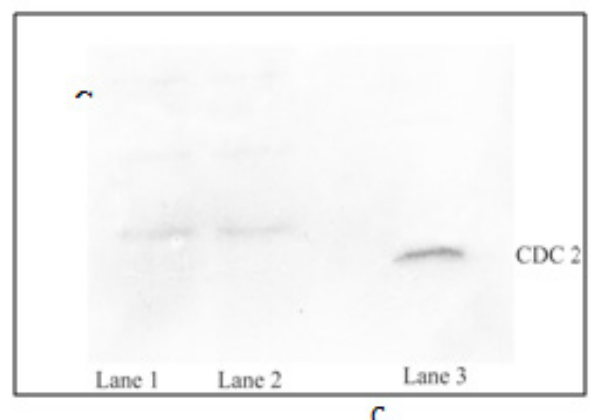

Figure 3: Reabsorption; Panel A: non-absorbed stained with Cdc2 antibody, \& Panel B: re-absorbed antibody incubated with Cdc2 peptide. Panel C: Lane 1 is protein extract from a young case, lane 2 is from an older case and lane 3 is purified Cdc2 protein. 
Table 4: Semi-quantitative assessment of pattern of Pin 1 immuno reactivity in Somatic and EW regions of Normal and AD cases.

\begin{tabular}{|c|c|c|c|c|}
\hline Case & $\begin{array}{c}\text { Somatic } \\
\text { NC }\end{array}$ & $\begin{array}{c}\text { Somatic } \\
\text { AD }\end{array}$ & $\begin{array}{c}\text { Edinger- } \\
\text { Westpha } \\
\text { INC }\end{array}$ & $\begin{array}{c}\text { Edinger- } \\
\text { Westphal } \\
\text { AD }\end{array}$ \\
\hline 1 & 3.9 & 2.3 & 1.8 & 1.0 \\
\hline 2 & 3.6 & 3.8 & 2.1 & 2.1 \\
\hline 3 & 3.4 & 3.4 & 1.9 & 1.4 \\
\hline 4 & 4.0 & 3.8 & 1.9 & 1.8 \\
\hline 5 & 3.6 & 3.5 & 1.0 & 1.0 \\
\hline
\end{tabular}

1: No reactivity

2: Light reactivity
3: Moderate reactivity

4: Strong reactivity

\section{Cyclin B1}

Having demonstrated that Cdc2 immunoreactivity appeared to be present in high levels in somatic cells of the NCNIII, we used a monoclonal antibody to cyclin B1 to determine if the potentially active $\mathrm{Cdc} 2 /$ cyclin B1 complex was present in the NCNIII. Consistent with the observations with respect to Cdc2, cyclin B1 immunoreactivity was present in somatic cells of both control and AD cases (Figure 4, panels A \& C respectively). Immunoreactivity was located primarily in the cell soma with the nucleus appearing devoid of staining. In contrast to somatic neurons, cyclin B1 immunoreactivity was substantially less intense in the EW. Stained EW neurons appeared ghost-like in both normal and AD cases. (Figure 4, B \& D).

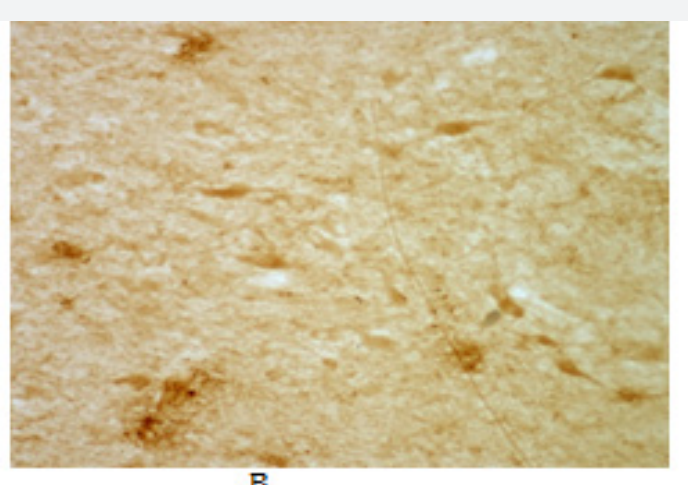

B

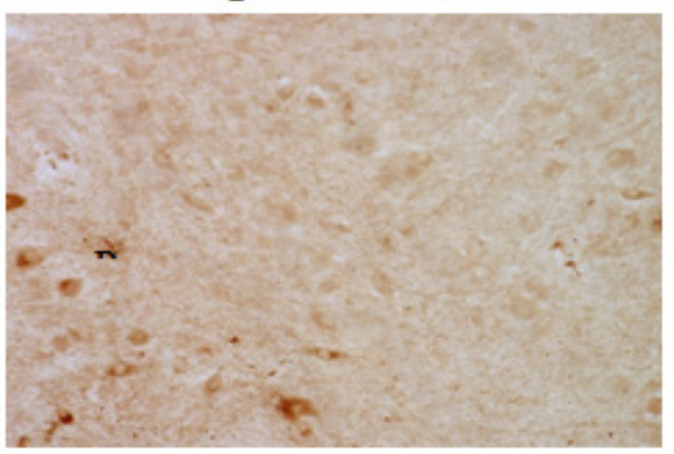

Figure 4: Cyclin B1: Panels A \& C somatic cells in normal case and AD case respectively. Panel $B$ and $D$ staining in EW of a normal case and $A D$ case.

\section{MPM-2}

It has been demonstrated that mitotic phosphoepitopes precede PHF pathology in the AD brain $[18,22]$. To determine if phosphoepitopes were present in NCNIII neurons as part of the phosphorylation cycle of tau either through the activity of Cdc2 or Cdk5, we used the monoclonal antibody MPM-2 to visualize such mitotic phosphoepitopes. MPM-2 is a universal marker for mitotic phosphoproteins that function in the M-phase. We hypothesized that such phosphoproteins would be activated in $\mathrm{AD}$ cases with PHF pathology but not in normal cases free of pathology. It has been reported that such phosphoepitopes are not present in differentiated neurons [22]. As shown in Figure 5 , phosphoepitopes recognized by MPM-2 were present in the nuclei of both EW (panel B) and somatic (panel A) neurons in normal cases. This tight compartmentalization was not seen in EW of AD cases (Figure 5, Panel D ). In AD cases, the EW neurons showed generalized staining for MPM-2 with some cells showing both cytoplasmic and nuclear staining. The pattern of staining was not unlike that observed with PHF-1 antibody to tau hyperphosphorylated epitopes and it is likely that MPM-2 is co-localized with such tau epitopes. In contrast, the somatic neurons of AD cases (panel C) showed similar nuclear staining as in normal cases. 


\section{Open Access Journal of Neurology \& Neurosurgery}

\section{Pin 1}

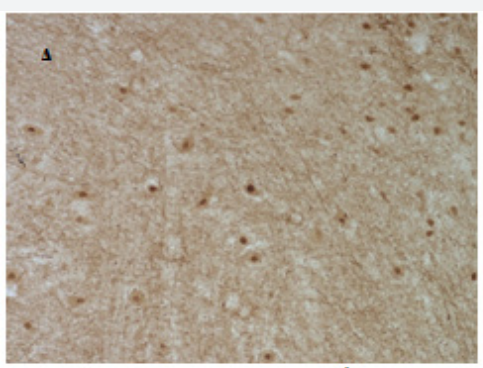

A

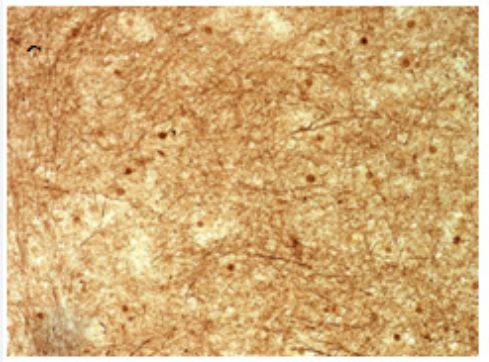

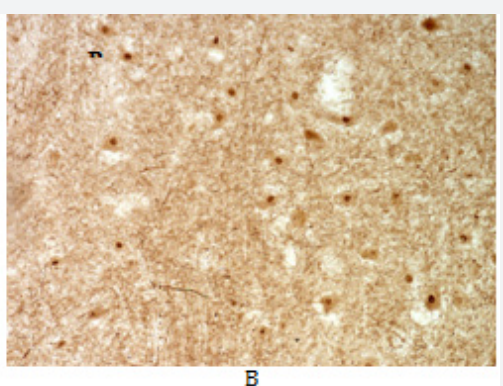

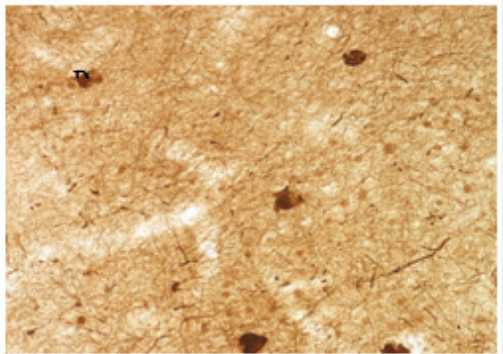

Figure 5: MPM-2: Panels A \& B, phosphoepitopes recognized by MPM-2 in EW cells (panels B \& D) and Somatic cells (panels A \& C) in normal cases and AD cases.

Using two monoclonal Pin1 antibodies (one monoclonal the generous gift of Dr. Ping Lu) we studied the cellular expression of Pin 1. As we hypothesized there were differences in the expression of Pin 1 in the EW and somatic cells of the AD cases (Figure 6, panel B). Expression of Pin 1, as evidenced by staining intensity, in EW cells compared to Somatic cells was substantially lower. We studied these same neuroanatomical regions in the normal control brains of young ( $\leq 50$ y.o.) and elderly ( $\geq 80$ y.o.) subjects free of any tangles and neuropil threads in the EW. The pattern of differences in Pin 1 expression (significently less in EW cells) were the same as observed in AD cases (Figure 6, panels D \& F). This later observation suggests that Pin1 may be present endogenously at far lower levels in the EW subdivision compared to the somatic portion. Lower levels of Pin1 in the EW may render it more vulnerable to abnormal hyperphosphorylation of tau perhaps explaining why this neuronal group is a selective target for such pathology compared to the somatic subdivision of the NCNIII.
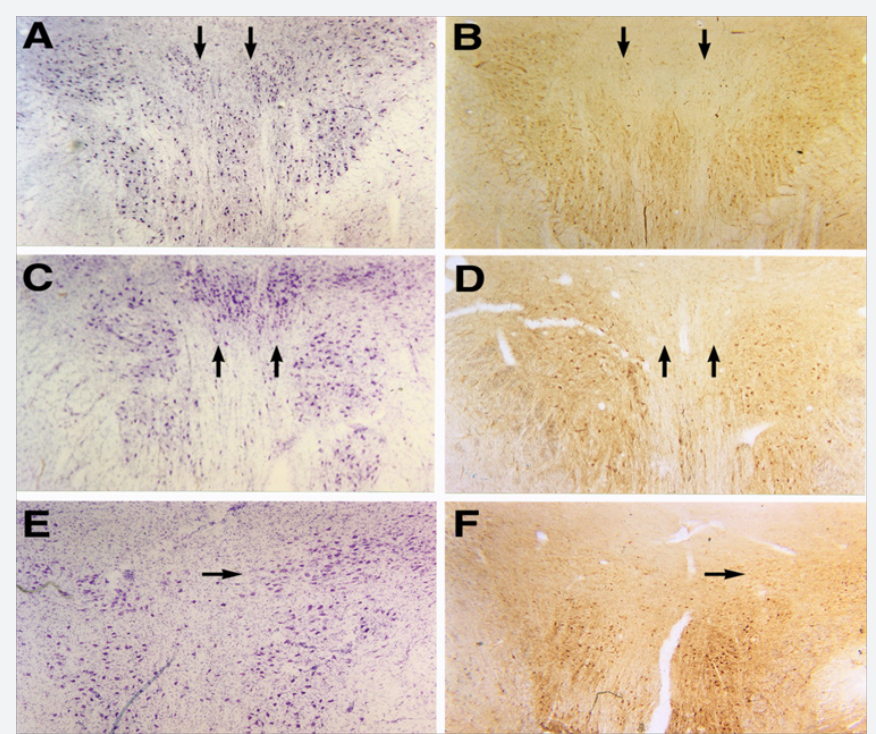

Figure 6: Pin 1: Panels A, C \& E Nissl stained sections containing both EW and somatic cell groups in the NCNIII from an AD brain, normal brain of a 50 year old and a normal brain from an 87 year old respectively. Panels B, D, F, contiguous sections stained for Pin 1 . Arrows indicate areas of lighter staining in the EW cell group in all brains. 
As with cdc2 we carried out a semi-quantitative analysis of the intensity of immunoreactivity in somatic and EW neurons. Our analysis revealed that there were no differences in staining intensity for either somatic or EW neurons for normal compared to $\mathrm{AD}$ cases (somatc, $\mathrm{p}=.343$; $\mathrm{EW} \mathrm{p}=.441$ ). Combining normal and AD subjects revealed that there were significant differences in staining intensity of somatic and EW neurons (somatic vs. EW, $\mathrm{p}=.000$ ). It is also noteworthy that the staining for somatic and EW neurons not only differed in intensity but also in distribution within the cellular compartment. Where EW neurons did exhibit faint staining the distribution was throughout the cell soma. In contrast in somatic neurons the strongest immunoreactivity was often seen in the peri-nucleur compartment.

\section{Discussion}

Preliminary work on patterns of cell vulnerability to pathology and death suggested that the NCNIII might serve as an ex-vivo model to explore the early vulnerability of some cell groups to tau pathology. Results from the present investigation demonstrate that key neurochemicals shown to be involved in the abnormal phosphorylation of tau are expressed differently in the somatic and EW subdivisions of the NCNIII. In EW neurons of normal cases Cdk5 immunoreactivity was considerably fainter with less perinuclear or nuclear staining compared to $\mathrm{AD}$ cases. The localizarion of Cdk5 in the EW of AD cases may represent its activation in EW neurons that exhibit heavy tau phosphorylation. In general we observed in normal cases that EW neurons stain less strongly for Cdk5 compared to neurons in $\mathrm{AD}$ cases. This observation is consistent with our finding of low Pin 1 immunoreactivity in these neurons.

The most surprising observation in this study was that Cdc2, thought to be normally down-regulated in terminally differentiated cells, was strongly present in the somatic portion of the NCNIII in both normal and AD cases. Some investigators have suggested [29], that part of the degenerative process in $\mathrm{AD}$ involves a mitotic process that is aborted early in its unfolding which would explain the presence of mitotic regulating proteins such as Cdc2 in pathologically damaged cells. However, the findings reported here suggest that some of these mitotic proteins are also present in high quantities in cells that are protected against the pathological damage of abnormal tau phosphorylation. The pattern of staining we observed, if confirmed with further studies, suggests that Cdc2 may play other than a mitotic regulating role in some terminally differentiated cells. It may, similarly to Pin 1, play a neuroprotective role. Our findings also suggest the possibility that the observations reported in other studies of staining for $\mathrm{Cdc} 2$ in degenerating neurons in $\mathrm{AD}$ may not represent the reentry of these cells into mitosis.

The association of stronger expression of Pin 1 with a protected neuronal population (somatic cells in NCNIII) suggests that this prolyl isomerase which has been shown to restore the function of phosphorylated tau might play a neuroprotective role alone or in concert with other proteins. We first suggested this possibility in preliminary work in our laboratory in 1999 [30]. It was suggested by some researchers [10] that Pin 1 might be sequestered by PHF-tau. Our initial observations which are confirmed here demonstrated that Pin 1 rather than being sequestered by PHF-tau is expressed at endogenously lower levels in cell populations (EW of NCNIII) that are subject to early and significant tau pathology and subsequent cell drop out. Rather than Pin 1 being sequestered by hyperphosphorylated tau and NFTs into an insoluble fraction, the endogenous absence of sufficient pools of Pin 1 may allow for a more robust process of tau hyperphosphorylation by Cdk5. As indicated above, Pin 1 regulates the biological function and dephosphorylation of MPM- 2 antigens. Pin 1 binds tau only when it is phosphorylated on Thr231. This in turn introduces a conformational change which restores tau's ability to bind microtubules and promote microtubule assembly. Pin 1 can also aid tau dephosphorylation by PP2A through indirect prolyl isomerization. There are likely many routes to cell aging and cell death. The work reported here suggests one possible mechanism for cell vulnerability due to aberrant tau phosphorylation.

An important observation and conclusion in this study was the striking and major difference in expression of epitopes that are of significance in the study of the pathological cascade of AD in EW and Somatic cell groups. These cholinergic cell groups (EW and Somatic) may provide a fruitful model and heuristic framework to explore the puzzling and largely unanswered question of selective cell vulnerability in $\mathrm{AD}$. Answering the question of selective cell vulnerability may well lead to critical insights and avenues to pursue in the development of new pharmacological therapies.

\section{References}

1. Foster NL, Chase TN, Mansi L, Brooks R, Fedio P, et al. (1984) Cortical abnormalities in Alzheimer's disease. Ann Neurol 16(6): 649-654.

2. Henderson VW, Finch CE (1989) The neurobiology of Alzheimer's disease . J Neurosurg 70(3): 335-353.

3. Scinto LF, Wu CK, Firla KM, Daffner KR, Saroff D (1999) Focal pathology in the Edinger-Westphal nucleus explains pupillary hypersensitivity in Alzheimer's disease. Acta Neuropathologica 97(6): 557-564.

4. Scinto LF, Frosch M, Wu CK, Daffner KR, Gedi N, et al. (2001) Selective cell loss in Edinger-Westphal in asymptomatic elders. Neurobiology of Aging 22(5): 729-736.

5. Davis DG, Schmitt FA, Wekstein DR, Markesbery WR (1999) Alzheimer neuropathologic alterations in aged cognitively normal subjects. J Neuropathol Exp Neurol 58(4): 376-388.

6. Hof PR, Glannakopoulos P, Bouras C (1996) The neuropathological changes associated with normal brain aging. Histopathology 11(4): 1075-1088.

7. Mukaetova-Ladinska EB, Harrington CR, Roth M, Wischik CM (1996) Alterations in tau protein metabolism during normal aging. Dementia 7(2): 95-103. 


\section{Open Access Journal of Neurology \& Neurosurgery}

8. Price JL, Davis PB, Morris JC, White DL (1991) The distribution of tangles, plaques and related immunohistochemical markers in healthy aging and Alzheimer's disease. Neurobiol Aging 12(4): 295-312.

9. Uboga NV, Price JL (2000) Formation of diffuse and fibrillar tangles in aging and early Alzheimer's disease. Neurobiol Aging 21(1): 1-10.

10. Lu PJ, Wulf G, Zhou XZ, Davies P, Lu KP (1999) The prolyl isomerase Pin 1 restores the function of Alzheimer-associated phosphorylated tau protein. Nature 399(6738): 784-788.

11. Gustke N, Steiner B, Mandelkow EM, Biernat J, Meyer HE, et al. (1992) The Alzheimer-like phosphorylation of tau protein reduces microtubule binding and involves Ser-Pro and Thr-Pro motifs. Federation of European Biochemical Societies 307(2): 199-205.

12. Lu Kun Ping, Liou Yih-Cherng, Vincent Inez (2003) Proline directed phosphorylation and isomerization in mitotic regulation and Alzheimer's disease. Bio Essays 25(2): 174-181.

13. Alonso AC, Zaidi T, Grundke-Iqbal I, Iqbal K (1994) Role of abnormally phosphorylated tau in the breakdown of microtubules in Alheimer's disease. Neurobiology 91(12): 5562-5566.

14. Haroutunian V, Purohit D, Perl DP, Marina D, Khan K, et al. (1999) Neurofibrillary tangles in nondemented elderly subjects and mild alzheimer's disease. Arch Neurol 56(6): 713-718.

15. Price JL (1993) The relationship between tangle and plaque formation during healthy aging and mild dementia. Neurobiol Aging 14(6): 661663

16. Goedert M (1999) Pinning down phosphorylated tau. Nature 399: 739788

17. Singh TJ, Zaidi T, Grundke-Iqbal I, Iqbal K (1996) Non-prolinedependent protein kinases phosphorylate several sites found in tau from Alzheimer disease brain. Mol Cell Biochem 154(2): 143-151.

18. Vincent I, Jicha G, Rosado M, Dickson DW (1997) Aberrant expression of mitotic Cdc2/cyclin B1 kinase in degenerating neurons of Alzheimer disease brain. J Neurosci 17(10): 3588-3598

19. Dranovsky A, Vincent I, Gregori L, Schwartzman A, Colflesh D, et al (2001) Cdc2 phosphorylation of nucleolin demarcates mitotic stages and Alzheimer's disease pathology. Neurobiol Aging 22(4): 517-528.

20. Pei JJ, Grundke-Iqbal I, Iqbal K, Bogdanovic N, Winblad B, et al. (1998) Accumulation of cyclin-dependent kinase 5 ( Cdk5) in neurons with early stages of Alzheimer's disease neurofibrillary degeneration. Brain Res 797(2): 267-277.

21. Wada Y, Ishiguro K, Itoh TJ, Uchida T, Hotani H, Saito T, Kishimoto T, Hisanaga S. (1998). Microtubule-stimulated phosphorylation of tau at Ser202 and Thr205 by Cdk5 decreases its microtubule nucleation activity. J Biochem 124(4): 738-746.

22. Husseman JW, Nochlin D, Vincent I (2000) Mitotic activation: a convertgent mechanism for a cohort of neurodegenerative diseases. Neurobiol Aging 21(6): 815-828

23. Peter M, Nakagawa J, Doree M, Labbe JC, Nigg EA (1990) Identification of major nucleolar proteins as candidate mitotic substrates of Cdc2 kinase. Cell 60(5): 791-801.

24. Mckhann G, Drachman D, Folstein M, Katzman R, Price D, et al. (1981) Clinical diagnosis of Alzheimer's disease: report of the NINCDS-ADRDA Work Group under the auspices of Department of Health and Human Services Task Force on Alzheimer's Disease. Neurology 34(7): 939944.

25. Khachaturian Z (1985) Diagnosis of Alzheimer's disease. Arch Neurol 42(11): 1097-1105.

26. Mirra SS, Heyman A, McKeel D, Sumi SM, Crain BJ, et al. (1991) The Consortium to Establish a Registry for Alzheimer's Disease (CERAD). Part II. Standardization of the neuropathologic assessment of Alzheimer's disease. Neurology 41(4): 479-486.

27. Lee MS, Kwon YT, Li M, Peng J, Friedlander RM, et al. (2000) Neurotoxicity induces cleavage pf p35 to p25 by caplain. Nature 405(6784): 360-364

28. Tsujioka Y, Takahashi M, Tsuboi Y, Yamamoto T, Yamada T (1999) Localization and expression of Cdc2 and cdk4 in Alzheimer brain tissue. Dement Geriatr Cogn Disord10(3): 192-198.

29. Vincent I, Zheng JH, Dickson D, Kress Y, Davies P, et al. (1998) Mitotic phosphoepitopes precede paired helical filaments in Alzheimer's disease. Neurobiology of Aging 19(4): 287-296.

30. Scinto LFM, Daffner K, Peck M, Langenkamp L, Bu J, et al. (2002) Neurochemical differences and cell loss in the somatic and EdingerWestphal divisions of cranial nerve III. Neurobiology of Aging 23: S491.

\section{Your next submission with Juniper Publishers will reach you the below assets}

- Quality Editorial service

- Swift Peer Review

- Reprints availability

- E-prints Service

- Manuscript Podcast for convenient understanding

- Global attainment for your research

- Manuscript accessibility in different formats

( Pdf, E-pub, Full Text, Audio)

- Unceasing customer service

Track the below URL for one-step submission

https://juniperpublishers.com/online-submission.php 